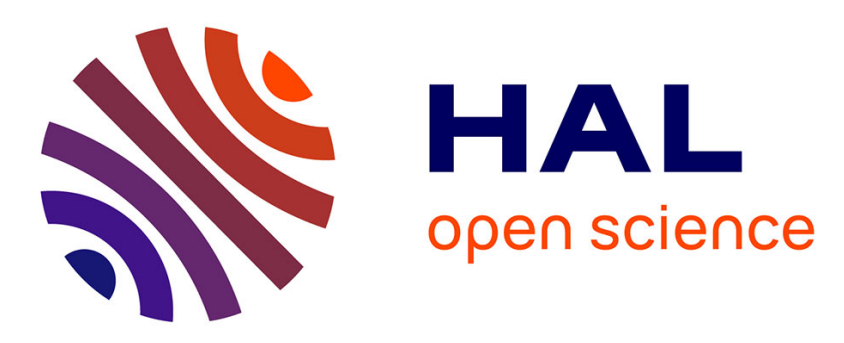

\title{
Evaluation of energy aware routing metrics for RPL
}

\author{
Lilia Lassouaoui, Stephane Rovedakis, Francoise Sailhan, Anne Wei
}

\section{To cite this version:}

Lilia Lassouaoui, Stephane Rovedakis, Francoise Sailhan, Anne Wei. Evaluation of energy aware routing metrics for RPL. 12th International Conference on Wireless and Mobile Computing (WiMob), Oct 2016, New York, United States. 10.1109/WiMOB.2016.7763212 . hal-02468579

\section{HAL Id: hal-02468579 \\ https://hal.science/hal-02468579}

Submitted on 26 Nov 2020

HAL is a multi-disciplinary open access archive for the deposit and dissemination of scientific research documents, whether they are published or not. The documents may come from teaching and research institutions in France or abroad, or from public or private research centers.
L'archive ouverte pluridisciplinaire HAL, est destinée au dépôt et à la diffusion de documents scientifiques de niveau recherche, publiés ou non, émanant des établissements d'enseignement et de recherche français ou étrangers, des laboratoires publics ou privés. 


\title{
Evaluation of Energy Aware Routing Metrics for RPL
}

\author{
Lilia Lassouaoui, Stephane Rovedakis, Françoise Sailhan, Anne Wei \\ CEDRIC Laboratory, \\ Conservatoire National des Arts et Métiers, \\ 292 rue Saint Martin, 75003 Paris, FRANCE \\ Email: firstname.name@cnam.fr
}

\begin{abstract}
In the past few years, the Internet of Things is driving the need for extending the Internet to constrained devices, including sensors and actuators. The IPv6 Routing Protocol for Low power and lossy networks (RPL) is appearing as an emerging IETF standard especially tailored for Low Power Area Networks (6LoWPAN). RPL constructs a Direct Acyclic Graph (DAG) according to an objective function that governs the routing according to some metric(s) and constraint(s). In the last decade, several metrics and constraints have been proposed, but for the to best of our knowledge this is the first comparative evaluation of RPL energy-aware routing metrics. In this paper, we survey energy-aware routing metrics. Then, we evaluate the performance of each, considering grid and random topologies. Moreover, we consider in this evaluation two models for the exchange of messages: a model with no packet loss and a second one with $40 \%$ of packet loss. Our experiments show that multi-criteria metrics outperform other metrics.
\end{abstract}

Keywords-RPL; energy-aware routing metrics; evaluation.

\section{INTRODUCTION}

Wireless Sensor Networks (WSNs) remain an emerging technology that has a wide range of applications including environmental monitoring, smart space and robotic exploration. WSN are characterised by constrained nodes with limited processing capabilities and memory, which are typically battery-operated and interconnected by wireless links that are operating at a low data rate. WSN are usually experiencing a high loss rate coming from the low power and lossy nature of the links. Such constraints combined with a typical large number of sensors have posed many challenges related to the configuration, management and routing. In order to tackle this issue, the IETF has standardised RPL [1], a new IPv6 routing protocol especially taylored for Low power and Lossy Networks (LLN). In compliance with the IPv6 over Low power Wireless Personal Area Networks (6LoWPAN) standard, RPL supports the idea of applying IPv6 [2] even to the smallest device by providing a mechanism whereby multipoints-to-point traffic from sensors inside the $6 \mathrm{LoW}$ PAN network towards a central control point (e.g., a server on the Internet) as well as point-to-multipoint traffic from the central control point to the sensors inside the 6LoPWAN are enabled. Support for point-to-point traffic is also available. For this purpose a Destination Oriented Directed Acyclic
Graph (DODAG) is built. This DODAG is constructed using an objective function which defines how the routing metric is calculated. In particular, this objective function determines how routing contraints and metrics are taken into account to determine the best route.

Qasem et al. [3] compared the performance of the two objective functions OF0 and MRHOF (see Section II-A for more details), which are available by default in RPL. In this evaluation, the authors consider a random and grid topology of different size (20, 30, 40 and 45 nodes). Moreover, they have evaluated the performance of these two objective functions with different packet reception ratios: no packet loss or $40 \%$ of packet loss. Their conlusions emphasized that MRHOF outperforms OF0 for most of the considered scenarii in random and grid topology. During the last decade, several metrics and constraints have been considered (see Section II-A for a detailed survey).

In this paper, we compare the performance of several RPL routing metrics proposed for saving power and maximizing lifetimes. To the best of our knowledge, this is the first comparative study with as many RPL routing metrics. We further perform an evaluation considering two network topologies, namely a grid topology and a random network. For each topology, we consider a perfect model with no packet loss and a more realistic model with $40 \%$ of packet loss. We conduct our experiments on top of the Cooja simulator [4], using Contiki OS 3.0. Simulation results show that multicriteria metrics perform better.

The remainder of this paper is organized as follows. We first provide in Section II an overview of RPL and its related metrics. Then, we present an evaluation of the performance of energy-aware routing metrics (Section III). We conclude this article with a summary of our contribution along future work.

\section{BACKGROUND}

The Routing Protocol for Low power and lossy network (RPL) [1] has been proposed by the IETF Routing Over Low power and Lossy networks (ROLL) working group. RPL is a distance-vector routing protocol targeting IPv6 networks. In compliance with the IPv6 architecture, it builds 
a Directed Acyclic Graph (DAG) so as to establish bidirectional routes between sensors. RPL is mainly designed to exchange data between each (RPL) node and a particular node, called sink node. The sink node acts as a common transit point that bridges the LLN with the IPv6 networks. It also represents a final destination node. The traffic flows supported by RPL, include sensors-to-sink, sensor-to-sensor, sink-to-sensors. A sensor network can be used for different applications and several sink nodes can coexist, i.e., we can have potentially one sink per application. A Destination Oriented DAG (DODAG) is constructed for each application according to a specific function (called Objective Function) which optimizes a specified metric for data routing, e.g., minimizes the network distance. Every DODAG is rooted at the corresponding application sink (DODAG root). Some applications can optimize objective function, which may be contradictory with another application. To this end, the concept of RPL instance has been introduced. A RPL instance brings together a subset of DODAGs in a sensor network which follow the same objective function. Several RPL instances can run concurrently, but a node belongs to at most one DODAG per RPL instance.

RPL separates packet processing and forwarding from the routing optimisation functions which may include minimising energy, latency and generally speaking satisfying constraints. In particular, RPL provisions routes towards the DODAG roots which is optimised with respects to the Objective function. In order to create and maintain a DODAG, RPL specifies a set of ICMPv6 control messages, such as DODAG Information Object (DIO) and DODAG Information Solicitation (DIS). The root starts the construction of the DODAG by broadcasting a DIO message carrying several parameters, including an affiliation with a DODAG (DODAGID), a rank which represents the position of the node with regards to the DODAG root, a routing cost and its related metrics, a Mode of Operation (MOP). The nodes that are in communication range with the root decide whether to join the DODAG or not. In particular, based on the neighbours ranks and according to the objective function, each node selects its DODAG parent. For this purpose, the node provision a routing table, for the destinations specified by the DIO message, via parent(s). Then, the node originates its own DIO message. Rather than waiting for the DIO message, node may also broadcast a DIS message requesting information from the other RPL nodes. Overall this DODAG root permits to support sensors-to-root traffic, which is a dominant flow in many applications.

Sensor-to-sensor traffic flows up toward a root and then down to the final destination (unless the destination is on the upward route). For this purpose, RPL establishes downward routes using Destination Advertisement Object (DAO) messages. DAO message is an optional feature. RPL supports two modes of Downward traffic: Non-Storing (fully source routed) or Storing (fully stateful). In the Non-Storing case, the packet travels towards the root before traveling Down ; the only device with a routing table is the root that acts as a router, hence source routing is used, i.e., the root indicates in the data packet the full route towards the destination. In the Storing case, sensors are configured as routers and maintain a routing table as well as a neighbour table that are used to look up routes to sensors. Thus, packet may be directed down towards the destination by a common ancestor of the source and the destination prior reaching a root.

In order to increase the network lifetime, RPL uses a dynamic dissemination algorithm, called Trickle. This algorithm adapts the rate at which DIO messages are sent by adjusting a timer. A DIO message is sent every Imin ms during the DODAG construction, and when the DODAG construction has converged this interval is doubled at each time period until reaching a maximum interval corresponding to $\operatorname{Imax} \mathrm{ms}$. When the DODAG reconfigurate due to e.g. the addition of new nodes or the detection of an inconsistency, RPL resets the timer to Imin. RPL also includes a mechanism to detect and suppress loops in the DODAG, based on the ranks in the DODAG. This loop-free property is obtained by insuring that the ranks increases in a strickly monotonically fashion, from the sink toward the leaf nodes. Therefore, every node compares the ranks of its neighbors to detect inconsistency, which is materialised by e.g., the reception of a downward data packet from a neighbor with a higher rank. When node detects a loop, it initiates a route poisoning (i.e., it broadcasts an infinite rank) so as to trigger a reconstruction of its sub-DODAG.

\section{A. Objective Functions}

An Objective Function (OF) specifies the objectives used to compute the (constrained) path and to select parents in DODAG. In practice, it defines the translation of metric(s) and constraint(s) into a value called Rank, which approximates the node distance from a DODAG root. Regardless of the particular OF used by a node, rank always increases so that loop-free paths are always formed. The definition of the OF is separated from the core RPL protocol. It allows RPL to meet different optimization criteria for a wide variety of applications. For a detailed survey on the OF, the interested reader may refer to [5]. The ROLL working group has specified two types of OFs: Objective Function zero (OF0) and Minimum Rank Hysteresis Objective Function (MRHOF). OF0 is the default objective function that uses the hop count as routing metric. The MRHOF minimizes the routing metric and uses the hysteresis mechanism to reduce the churn coming from small metric changes for a better path stability.

RPL supports constraint-based routing. A constraint may be applied to link or node, and, if a link/node does not satisfy the given constraint, it is pruned from the candidate neighbors set, hence leading to a constrained shortest path. 
A metric is used in association with an $\mathrm{OF}$ for route optimization. The ROLL working group proposes two types of metrics: the node metric and link metric. The node metric represents the node state, e.g., node energy or node hops. The Link metric reflects the route quality, e.g. latency, throughput, Expected Transmission count (ETX). These metrics can be additive or multiplicative, they can also refer to a maximum or minimum property along a path in the DODAG.

In order to construct and update the DODAG, each nonroot node has to select a preferred parent. This selection is performed by computing the path cost for each parent (neighbor with a lower Rank). The path cost is a numerical value which represents a property of the path toward the sink node. It is computed by summing up the selected node/link metric to the advertised path cost. The best cost returned by the $\mathrm{OF}$ using the specified metric for each candidate parent is used to select the preferred parent, i.e., the parent on the path with the best cost. The path cost is computed again either if the node/link metric is updated or if a new metric is advertised. When MRHOF is used, according to the hysteresis mechanism the current preferred parent is changed if the difference between the current and the new path cost is at least equal to a specified threshold.

After selecting its preferred parent $P$, a non-root node $q$ computes its rank $R(q)$ as follows: $R(q)=$ $R(P)+$ rank_increase, with $R(P)$ defining the Rank advertised by $P$ and rank_increase the rank increment. Note that a DODAG root advertises a Rank equal to rank_increase. The Rank and the path cost computed by each node are disseminated in a DIO message.

\section{B. Some Routing Metrics Proposed for RPL}

Several routing metrics have been proposed in the litterature to increase the network lifetime, to maximize the reliability or to minimize the latency. In this paper, we focus on the energy-aware routing metrics because the energy is a key criterion of wireless sensor networks.

One of the classical and popular routing metric available in several RPL implementations is the Expected Transmission count (ETX). ETX estimates the number of transmissions that take place through a link before the reception of a correct acknowledgment. This value can be computed as: $E T X=\frac{1}{P D R_{s \rightarrow d} \times P D R_{s \rightarrow d}}$, with $P D R_{d \rightarrow s}$ defining the estimated packet delivery ratio from $s$ to $d$. More particularly, this estimated packet delivery ratio is computed as the ratio between the number of transmitted packets and the number of acknowledged packets, including retransmission(s). Then, among the neighbours $N_{i}$, using MRHOF a node $i$ selects as preferred parent, the neighbour characterised by the minimum ETX, i.e., $\min _{j \in N_{i}} E T X_{j}$. The lower is ETX, the better is the link quality. The Rank $R(i)$ of node $i$ with preferred parent $P$ is given by: $R(i)=R(P)+$ rank_increase. It is disseminated by node $i$ using a DIO message. ETX seems to be a good candidate to reduce the end-to-end delay. Indeed, the lower is the retransmission number, the better is transmission time for a data packet toward the sink. In addition, since communication is the most energy consuming activity, ETX allows to reduce the energy consumed at each node. However, this does not permit to select a route composed of nodes with high battery level.

In order to design an energy-aware route selection, the residual energy ResEng $g_{i}$ can be used as a RPL metric. The residual energy is computed as the difference between the maximum battery level $M a x E n g_{i}$ and the energy consumed EngCons $s_{i}$ by a node $i$, i.e., ResEng ${ }_{i}=$ MaxEng $g_{i}-E_{n}$ Cons $s_{i}$. The energy consumed by a sensor is due to the computation and the radio communication (i.e., transmission and listening). Demicheli [6] proposed the first RPL metric which considers the energy consumed by sensor nodes along a path. The Rank $R(i)$ of each node $i$ is obtained by adding an increment (fixed to 16 by the author) to the Rank $R(P)$ of its preferred parent $P$, i.e., $R(i)=R(P)+$ rank_increase. Each node $i$ sends in DIO messages its Rank as well as the energy consumed along the path PathEngCons $(i)$ in the Metric Container field, with PathEngCons $(i)$ equals to the sum of PathEngCons $(P)$ sent by its preferred parent $P$ and $E_{\text {EngCons }}$. The preferred parent is the parent with the lowest energy consumed along the path. The main drawback is that a path toward the sink may contain a node with a very low residual energy. To tackle this issue, $\mathrm{Xu}$ et al. [7] and Kamgueu et al. [8] consider the residual energy as a routing metric. $\mathrm{Xu}$ et al. [7] have proposed to use RPL with a residual energy metric: the Rank $R(i)$ of node $i$ is equal to the Rank of the preferred parent $R(P)$ plus the residual energy ResEng R $_{i}$ of i.e., $R(i)=R(P)+R_{e s E n g_{i}}$. Each node selects as preferred parent the one with the highest Rank, and $i$ sends a DIO message with its Rank and an idle Metric Container field. Kamgueu et al. [8] define the cost of a path $P W_{i}$ of a node $i$ toward the sink as the minimum among the residual energies along the path. Therefore, each node sends in DIO messages its rank and its path cost using the Metric Container field. Every node $i$ computes the path cost that can be obtained for each parent (as the minimum between its residual energy and the path cost sent by the parent), and selects as preferred parent the one with the maximum computed path cost, i.e., $P W_{i}=\min \left(\max _{j \in N_{i}}\left\{P W_{j}\right\}\right.$, ResEng $\left.g_{i}\right)$, where $N_{i}$ refers to the neighbours of node $i$.

Some applications require data transmission with a low delay. Several routing metrics have been proposed to minimize the end-to-end delay with RPL [9], [10], [11]. Chang et al. [12] propose an energy-aware metric which considers the number of retransmissions. For this purpose, the residual energy is combined with the ETX. Each node $i$ sends its Rank and its residual energy ResEng $g_{i}$ using the Container Metric field in DIO messages. The preferred parent is selected 
Table I

SUMMARY OF THE PRESENTED RPL METRICS

\begin{tabular}{|c|c|c|c|c|}
\hline \multirow[b]{2}{*}{ Paper } & \multirow[b]{2}{*}{$\begin{array}{l}\text { Energy } \\
\text {-aware }\end{array}$} & \multicolumn{2}{|c|}{ Metrics information } & \multirow[b]{2}{*}{ Topology } \\
\hline & & Energy & $\begin{array}{c}\text { Link } \\
\text { quality }\end{array}$ & \\
\hline$[14]$ & No & - & Yes & $\begin{array}{c}\text { Grid } \\
\text { \& Random }\end{array}$ \\
\hline [6] & Yes & $\begin{array}{c}\text { Energy } \\
\text { consumption }\end{array}$ & No & $\begin{array}{c}\text { Grid } \\
\text { \& Random }\end{array}$ \\
\hline [7] & Yes & Residual energy & No & $\begin{array}{c}\text { Grid } \\
\text { \& Random }\end{array}$ \\
\hline [8] & Yes & Residual energy & No & $\begin{array}{c}\text { Grid } \\
\text { \& Random }\end{array}$ \\
\hline [12] & Yes & Residual energy & Yes & Random \\
\hline [13] & Yes & Residual energy & Yes & Random \\
\hline
\end{tabular}

among the parent $j$ of $i$ which gives the minimum of the weighted function: $\alpha \frac{E T X_{j}}{M a x \_E T X}+(1-\alpha) \times\left(1-\frac{R_{e s E n g_{i}}}{M a x E n g_{i}}\right)$, where Max_ETX and ${ }^{-}{ }_{\text {MaxEng }}$ are respectively the maximum ETX value of a link and the maximum battery level of $i$. The Rank of each node is computed as it is done by the ETX metric (and described above). Recently, Iova et al. [13] have proposed another routing metric, called Expected LifeTime (ELT), to better optimize the network lifetime. This metric takes into account the link quality, the residual energy and the traffic. First of all, each node $i$ computes its expected lifetime $E L T_{i}$ following Equation 1:

$$
E L T_{i}=\frac{\text { ResEng }_{i}}{T_{i} \times \frac{E T X(i, P)}{\text { Data_Rate }} \times \text { PowT } X_{i}},
$$

where $T_{i}$ is the traffic of $i$ (in bits/s), $\operatorname{ETX}(i, P)$ is the ETX value of the link to the preferred parent $P$ of $i$, Data_Rate is the rate at which data is sent (in bits/s) and PowT $X_{i}$ is the power consumed by a radio transmission made by $i$. For each path in the DODAG, the minimum expected lifetime is propagated along the path using the Metric Container field of DIO messages. Each node $i$ selects as preferred parent the one which gives the maximum expected lifetime, i.e., $\max _{j \in N_{i}} E L T_{j}$. The Rank associated to a node $i$ in the DODAG is computed as for ETX.

Tab. I presents a brief summary of the RPL metrics described in this section, it also gives the topology considered by the authors to evaluate their metrics.

\section{Metric Evaluation}

In this section, we present the evaluation we conducted for several RPL routing metrics described in the previous section.

\section{A. Simulation setup}

In order to simulate and analyze the performance of RPL, we use the Cooja simulator [4], a flexible Java-based simulator which supports $\mathrm{C}$ program language as the software design language by using Java Native Interface. We simulate a Wireless LLN consisting of 56 nodes which are emulated as Tmote sky mote [15] (a widely used sensor platform) with a $2.4 \mathrm{GHz} \mathrm{CC} 2420$ radio transceiver with
IEEE 802.15.4 operating at the radio layer. These nodes are deployed over a 300 x $300 \mathrm{~m}$ square. We consider two topologies: a grid and a random network. With the grid, the sink is located at the bottom right corner (Figure 1(a)). This sink location represents a worst case scenario (comparing to a sink located at the grid center): a higher congestion is observed around the sink because very few sensors are connected to the sink. Moreover, in this topology we have a mean density of 6.5 , half of the nodes have 8 neighbors while the other have 5 neighbors in the network (except the four nodes on the corners with 3 neighbors). With the random topology, nodes are randomly positioned in the $300 \times 300 \mathrm{~m}$ square (Figure 1(b)) with the sink (green node) located on the left. The density in this second network is smaller than in the grid network, the nodes in this second topology have three neighbors in average. In particular, the distance separating the nodes from the sink in the two topologies is given in Tab. II. We use the ContikiOS 3.0 with ContikiMac [16] which provides a power efficient medium access control by turning off the radio 99 percent of the time. We further rely on RPL as a routing protocol and we simulate a sensors-to-sink traffic wherein each node periodically sends to the sink some data packets, at a rate of 6 packets per minute, i.e., we consider Constant Bit Rate (CBR) convergecast flows. Note that each node starts sending its first data packet $65 \mathrm{~s}$ after the beginning of a simulation. The main parameters used during the simulation are summarized in Tab. III. The TX and RX rates define respectively the success ratios in transmission and reception mode. We consider two models for message transmission: (i) a first model with no packet loss used to better evaluate the performance of the metrics $(\mathrm{RX}=100 \%)$, and (ii) a second one with a success ratio in reception mode of $60 \%$ ( $\mathrm{RX}=60 \%$ which induces a packet loss of $40 \%$ ). We average the simulation results over 10 simulation runs, each one of 5 hours duration.

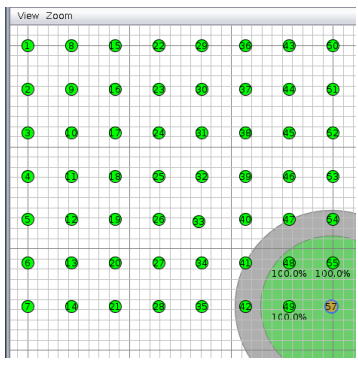

(a) Grid topology

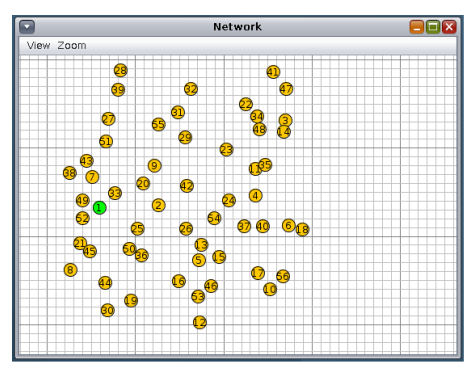

(b) Random topology
Figure 1. Grid and Random topology considered in the simulation. 
Table II

Node Distribution

\begin{tabular}{|c|c|}
\hline \multicolumn{2}{|c|}{ Grid Topology } \\
\hline Distance to the Sink & Number of Nodes \\
\hline $70 \mathrm{~m}$ & 3 \\
\hline $140 \mathrm{~m}$ & 5 \\
\hline $210 \mathrm{~m}$ & 7 \\
\hline $280 \mathrm{~m}$ & 9 \\
\hline $350 \mathrm{~m}$ & 11 \\
\hline $420 \mathrm{~m}$ & 13 \\
\hline $490 \mathrm{~m}$ & 7 \\
\hline \multicolumn{2}{|c|}{ Random Topology } \\
\hline Distance to the Sink & Number of Nodes \\
\hline $0-45 \mathrm{~m}$ & 4 \\
\hline $45 m-90 m$ & 14 \\
\hline $90 \mathrm{~m}-135 \mathrm{~m}$ & 9 \\
\hline $135 \mathrm{~m}-185 \mathrm{~m}$ & 11 \\
\hline $185 \mathrm{~m}-230 \mathrm{~m}$ & 6 \\
\hline $230 m-275 m$ & 8 \\
\hline $275 \mathrm{~m}$ & 3 \\
\hline
\end{tabular}

Table III

SIMULATION PARAMETERS

\begin{tabular}{|c|c|}
\hline Parametres & Value \\
\hline OF & MRHOF \\
RPL MOP & NO_DOWNWARD_ROUTE \\
Start Delay & $65 \mathrm{~s}$ \\
Imin & $2^{12} \mathrm{~ms}$ \\
Imax & $2^{20} \mathrm{~ms}$ \\
Data sent interval & $6 \mathrm{pkt} / \mathrm{min}$ \\
TX ratio & $100 \%$ \\
RX ratios & $100 \%$ and $60 \%$ \\
TX Range & $45 \mathrm{~m}$ \\
Interference Range & $70 \mathrm{~m}$ \\
\hline
\end{tabular}

\section{B. Evaluation criteria}

In this study, we evaluate the influence of the metrics in terms of energy consumption, Packet Delivery Ratio (PDR), end-to-end delay and number of control messages exchanged. We also consider two topologies: a grid and a random network.

- Energy Consumption - In order to compute the energy consumption, we rely on the Power-trace mechanism [17] provided by Contiki. The power-trace estimates the power consumption due to the CPU usage and the network-level activitities including packet transmission and reception. During our experiments, we focus on the period of time the radio is on. We further calculate the energy consumption $E_{i}$ at each node $i$ (in $\mathrm{mJ})$ :

$E_{i}=\frac{\left(T_{C P U} * I_{C P U}+T_{R X} * I_{R X}+T_{T X} * I_{T X}\right) * V}{R_{\text {timer }}}$

where $V$ corresponds to the battery voltage $(=3.6 \mathrm{~V})$, $I_{C P U} \quad(=1.8 \mathrm{mAh}), \quad I_{R X} \quad(=20 \mathrm{mAh}) \quad$ and $\quad I_{T X}$ $(=17.7 \mathrm{mAh})$ represent the current that has been consumed respectively during the CPU run time $T_{C P U}$, the radio listen run time $T_{R X}$ and the radio transmit run time $T_{T X}$ (all expressed in ticks). $R_{\text {timer }}$ represents the number of ticks per second $(=32768$ ticks/s).

- Packet Delivery Ratio (PDR) is defined as the number of packets that are successfully received by the sink, divided by the number of packets sent by all the nodes to the sink.

- End-to-end delay is defined by the period of time between the packet generation by the node source in the application layer and its reception by the sink (in the application layer).

- Control messages - In order to reflect the cost and stability of RPL network topology, we trace the number of control messages (i.e., DIS and DIO messages) exchanged in the network.

\section{Results}

In the following, we present our results. In particular, we consider the five following well known RPL metrics (that have been surveyed in Section II-B) used to optimize the network lifetime:

- ETX: this is the default metric for RPL, which considers the number of retransmissions for each link.

- Energy consumption: we consider the metric proposed by Demicheli [6] in which the path cost represents the sum of the consumed energies, called ENG-TOT hereafter.

- Residual energy: we consider the metric proposed by Kamgueu et al. [8] wherein the path cost is given by the minimum residual energy on the path, called $E N G$ MinMax hereafter.

- Residual energy + ETX: we selected the metric proposed by Chang et al. [12] in which the path cost is equal to a weighted function integrating ETX and the residual energy, called $R$ hereafter. The two parameters have the same weight in our simulation, i.e., we defined $\alpha=0.5$.

- Expected lifetime: we choose the metric proposed by Iova et al. [13] because it carrefully models the network lifetime, called ELT hereafter. However, we do not implemented the expected traffic associated to each node, since it requires to exchange additional control messages to estimate the traffic in the sub-DODAG of each node.

Energy consumption: We consider first the energy consumed by each routing metric (Figure 2). In our simulation, the nodes have the same initial battery charge of $853 \mathrm{mAh}$. For a better usage with the energy-aware routing metrics, we have represented this charge in Cooja on a scale of 255 (as suggested in the RPL standard) and every step of $3.345 \mathrm{mAh}$ decreases the battery level by one. In Figure 2, the percentage of time the radio is on mostly reflects the 


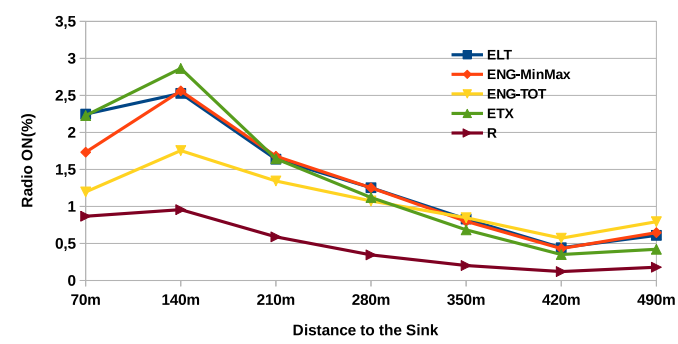

(a) Grid topology with $\mathrm{RX}=100 \%$

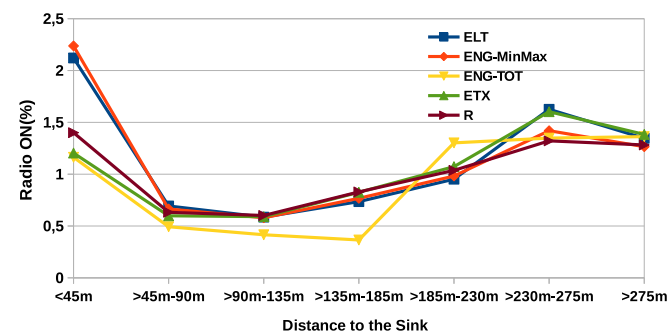

(c) Random topology with $\mathrm{RX}=100 \%$

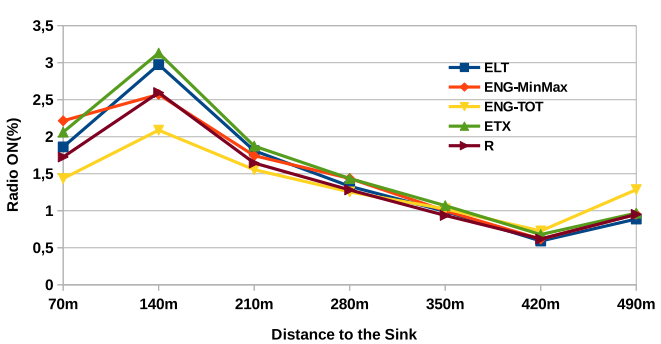

(b) Grid topology with $\mathrm{RX}=60 \%$

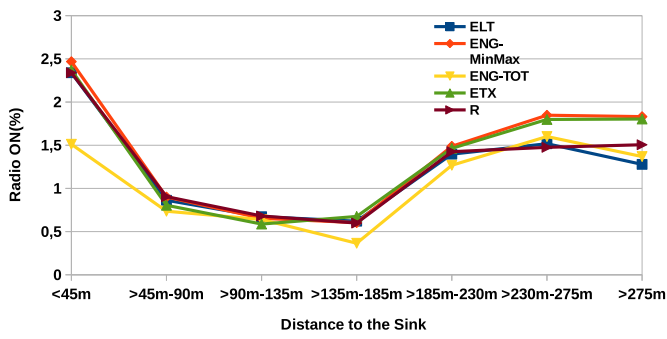

(d) Random topology with $\mathrm{RX}=60 \%$

Figure 2. Energy Consumption.

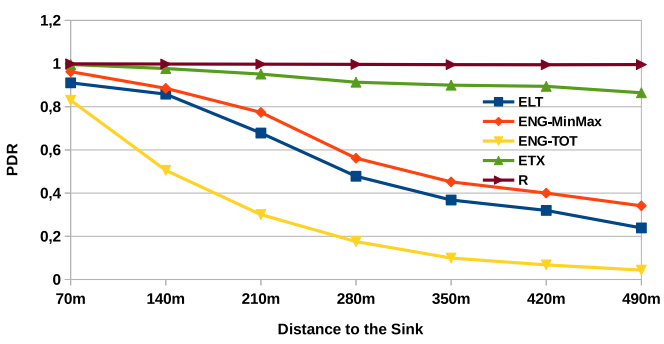

(a) Grid topology with $\mathrm{RX}=100 \%$

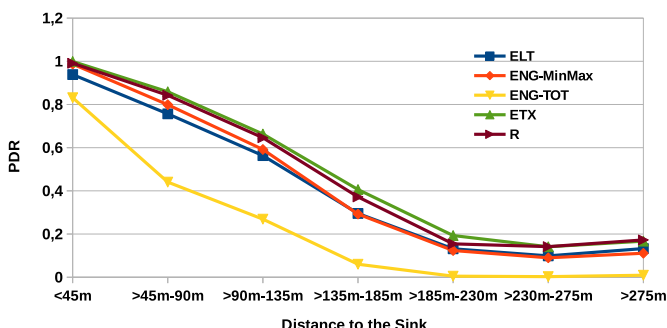

(c) Random topology with $\mathrm{RX}=100 \%$

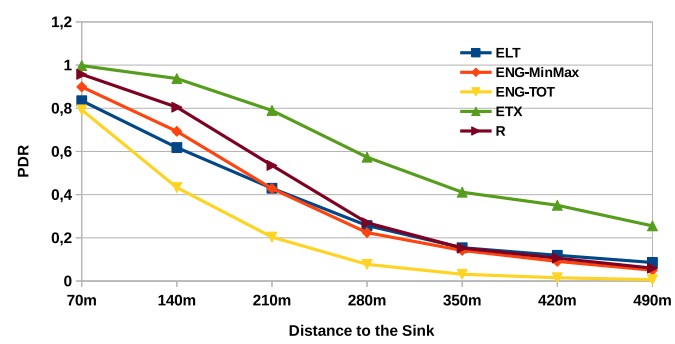

(b) Grid topology with $\mathrm{RX}=60 \%$

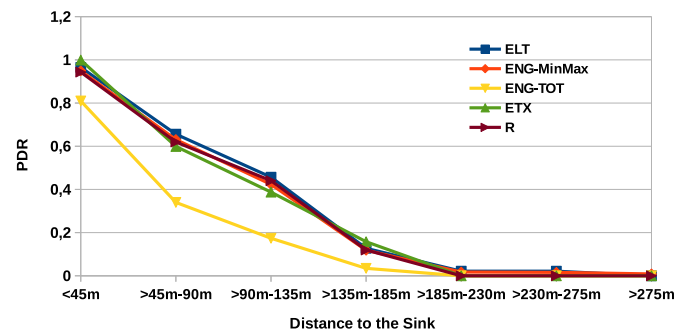

(d) Random topology with $\mathrm{RX}=60 \%$

Figure 3. Packet Delivery Ratio. 
energy consumed by the RPL protocol. In a grid topology with $\mathrm{RX}=100 \%$ (Figure 2(a)), the energy consumption increases and then decreases as a function of the distance to the sink for the five metrics. This increase is due to the fact that the sink represents a bottleneck. Then, as expected the energy consumption decreases as a function of the node distance. ETX is the routing metric which has the highest energy consumption around the sink, followed by the other energy-aware metrics ENG-MinMax and ELT, then ENGTOT and R. However, for the farthest nodes, the energy consumed by the ETX metric is lower comparing to the ENG-MinMax, ELT and ENG-TOT metrics due to the low retransmission rates (as reflected in Figure 3(a)). The R metric achieves the lowest energy consumption, the radio is on at most 1 percent of the time. In a grid topology with $\mathrm{RX}=60 \%$ (Figure 2(b)), the five metrics behave similarly as for the case $\mathrm{RX}=100 \%$. We observe a slight increase of the energy consumption which comes from the increased number of retransmissions. Moreover, the $\mathrm{R}$ metric achieves similar results as other energy-aware metrics contrary to the case $\mathrm{RX}=100 \%$. The $\mathrm{R}$ metric gives best results in the first half of the network and then we obtain similar results for all the metrics for the second half of the network.

With a random topology (Figures 2(c) and 2(d)), we observe the same behaviour when $\mathrm{RX}=100 \%$ or $\mathrm{RX}=60 \%$. The energy consumption is a little higher in the latter case because of packet retransmissions. The energy consumption decreases and then increases as a function of the distance to the sink for the five metrics. A higher energy consumption around the sink is due to a bottleneck; it decreases until a certain distance to the sink $(135 \mathrm{~m})$, then increases due to the packets loss (as indicated by PDR in Figures 3(c) and 3(d)). For the two topologies, we observe a low energy consumption for ENG-TOT metric comparing to the other metrics but it is a side effect of the low network load caused by a low PDR (as explained below for the PDR criteria).

Tab. IV presents for each metric the percentage of energy consumed after 5 hours of simulations and an extrapolation of the network lifetime expressed in days. It is noteworthy that the short network lifetime is related to the low initial battery charge (of $853 \mathrm{mAh}$ instead of $2000 \mathrm{mAh}$ in real Tmote sky mote platform [18]). We observe that the $\mathrm{R}$ metric outperforms all the metrics on grid topology with $\mathrm{RX}=100 \%$. It achieves a lifetime of 133 days, around 5 to 7 times better than the other energy-aware metrics. All the other metrics have a similar network lifetime (except ENGTOT). With RX $=60 \%$ on grid topology, we observe that the $\mathrm{R}$ metric achieves a lifetime equivalent to the other energyaware metrics. This is due to the packets retransmissions resulting from the high packet loss. On random topology with $\mathrm{RX}=100 \%$ or $\mathrm{RX}=60 \%$, all the metrics (except ENGTOT) give similar network lifetimes (from 10 to 13 days). We see half values in comparison with grid topology. This is a consequence of the packet retransmissions because of
Table IV

METRICS LifETIME

\begin{tabular}{|c|c|c|c|c|c|}
\hline \multicolumn{7}{|c|}{ Grid Topology } \\
\hline & ENG-MinMax & ENG-TOT & ETX & ELT & $\mathrm{R}$ \\
\hline $\mathrm{RX}=$ & $1.12 \%$ & $0.38 \%$ & $1.15 \%$ & $0.97 \%$ & $0.157 \%$ \\
\cline { 2 - 6 } $100 \%$ & $124698 \mathrm{mj}$ & $42212 \mathrm{mj}$ & $166098 \mathrm{mj}$ & $107822 \mathrm{mj}$ & $17359 \mathrm{mj}$ \\
\cline { 2 - 6 } & 19 days & 55 days & 18 days & 22 days & 133 days \\
\hline $\mathrm{RX}=$ & $0.81 \%$ & $0.40 \%$ & $1.37 \%$ & $0.82 \%$ & $0.93 \%$ \\
\cline { 2 - 6 } $60 \%$ & $88630 \mathrm{mj}$ & $43803 \mathrm{mj}$ & $151250 \mathrm{mj}$ & $91583 \mathrm{mj}$ & $103099 \mathrm{mj}$ \\
\cline { 2 - 6 } & 26 days & 52 days & 15 days & 25 days & 23 days \\
\hline \hline \multicolumn{6}{|c|}{ Random Topology } \\
\hline $\mathrm{RX}=$ & $1.77 \%$ & $0.71 \%$ & $1.71 \%$ & $1.71 \%$ & $1.78 \%$ \\
\cline { 2 - 6 } $100 \%$ & $196499 \mathrm{mj}$ & $78502 \mathrm{mj}$ & $188999 \mathrm{mj}$ & $189759 \mathrm{mj}$ & $196700 \mathrm{mj}$ \\
\cline { 2 - 6 } & 11 days & 29 days & 13 days & 13 days & 12 days \\
\hline $\mathrm{RX}=$ & $1.96 \%$ & $0.84 \%$ & $1.98 \%$ & $1.95 \%$ & $1.91 \%$ \\
\cline { 2 - 6 } $60 \%$ & $219994 \mathrm{mj}$ & $93744 \mathrm{mj}$ & $220104 \mathrm{mj}$ & $215300 \mathrm{mj}$ & $211666 \mathrm{mj}$ \\
\cline { 2 - 6 } & 10 days & 24 days & 10 days & 11 days & 11 days \\
\hline
\end{tabular}

a low PDR induced by the low average network density of 3 (see PDR criteria below). As noticed before, ENG-TOT metric has good network lifetimes generally, but it is a side effect of the low PDR reached.

Packet Delivery Ratio: In Figure 3, the Packet Delivery Ratio (PDR) decreases as a function of the distance to the sink for both the grid and random networks. Globally ETX metric performs well because it takes into account the link quality so as to choose the best parent, while ENG-TOT metric which ignores the link quality exhibits the worse PDR. Note that in a grid topology with RX $=100 \%$ (Figure $3(\mathrm{a})$ ), the best results are given by the R metric with a PDR close to 100 percent for any node. Better results are achieved by the $\mathrm{R}$ metric, which takes into account both the link quality and the residual energy, contrary to ETX metric for which a non neglectable amount of packets is lost due to the exhausted battery. The ENG-MinMax and ELT energy-aware metrics achieve a lower PDR from 100 percent near the sink to 30 percent for the farthest nodes, ENG-TOT gives the poorer results. For RX=60\% on grid topology (Figure 3(b)), we observe the same tendency for all the metrics but with smaller PDR values than for $\mathrm{RX}=100 \%$. We can notice a reversal between ETX and R metrics. R metric achieves a lower PDR than ETX metric, but it gives a little better values than other energy-aware metrics (except ENG-TOT). ETX metric has the highest PDR from 100 percent near the sink to 25 percent for the farthest nodes. With a random topology (Figures 3(c) and 3(d)), the packet delivery ratio decreases faster as fewer routes are present (the density of neighbours in transmission range being around 3). All the metrics achieve nearly the same PDR values. For RX=100\%, the values spread from 100 percent near the sink to 15 percent for the farthest nodes, while for $\mathrm{RX}=60 \%$ the PDR values are sharper with values between 100 and 15 percent for the first half of the network (until 135m) and then no data packet sent by a sensor in the second half of the network 
(distance higher than $135 \mathrm{~m}$ ) has been received by the sink. We can notice that ENG-TOT metric achieves the poorer PDR in all the cases by far.

End-to-end delay: The latency naturally increases along with the distance to the sink (Figure 4). In grid topology with $\mathrm{RX}=100 \%$ (Figure $4(\mathrm{a})$ ), the best end-to-end delay over all metrics is again obtained by the $\mathrm{R}$ metric with an end-to-end delay smaller than $200 \mathrm{~ms}$ for the farthest nodes and $100 \mathrm{~ms}$ for others. The obtained results are in agreement with the PDR values described previously. It is up to 5 times better than the worst delay achieved by the ENG-MinMax metric. The $\mathrm{R}$ metric is then followed by ETX metric which takes into account the link quality only. ETX metric has delay 3 times higher than R metric. For the energy-aware metrics, ENG-MinMax metric gives the poorer results with a delay between 300 and $500 \mathrm{~ms}$ for most of the nodes except for the farthest nodes whose packets are transmitted with $900 \mathrm{~ms}$ delay. ELT metric is situated between ENG-TOT and ENG-MinMax metrics. In the same topology with RX=60\% (Figure 4(b)), ENG-TOT metric achieves the lowest delay due to the low amount of packets to route unlike other metrics (see PDR values in Figure 3(b)). R metric looses its effectiveness compared to the case when $\mathrm{RX}=100 \%$, since the same weight is provided for the link quality and residual energy. Still, it remains close to the ETX metric. For the Random network with RX $=100 \%$ (Figure 4(c)), one may see that a sligh increase is followed by a sharp increase that is correlated with the high number of retransmissions due to a low PDR (as shown in Figure 3(c)). All the metrics (except ENG-TOT) gives similar results. With a lower RX of $60 \%$, the latency increases in the first half of the network for all the five metrics until reaching a maximum of $350 \mathrm{~ms}$ for ELT and $450 \mathrm{~ms}$ for other metrics (except ENG-TOT) for nodes at $135 \mathrm{~m}$. Then, the delay is null in the second half of the network because all the packets are dropped: at this stage, the reliability of RPL is involved. We can notice that, except ENG-TOT which gives the lowest delay in all the cases because of packet loss, ELT metric achieves in this case the lowest latency among all the metrics in the first half of the network from 10 to $100 \mathrm{~ms}$ better.

Control messages: The overhead expressed as the amount of control messages sent by RPL increases slowly as a function of the distance to the sink (Figure 5). In all the cases, we observe that ENG-TOT performs poorly; the overhead caused by ELT and ENG-MinMax metrics is relatively stable. The high amount of control messages for ENG-TOT metric is related to the low PDR achieved by this metric. In fact, this high amount of control messages exchanged results from route instabilities in the DODAG. This has been analyzed by Boubekeur et al. [19] which address this problem by reducing the maximum number of children that a node can have in RPL. We note that, appart from ENG-TOT metric, the control traffic is negligible for all the other metrics in the different simulation scenarii compared to the data traffic and as the DODAG stabilizes the control traffic decreases significantly.

Summary of the Experiments: On the whole, ETX metric achieves good results in the two types of topology in terms of PDR and delay. In counterpart, the energy-aware metrics show good results for energy consumption but poorer performance on PDR and delay because they do not take into account the link quality. ENG-TOT metric is a special case, it reaches a low energy consumption in comparison to the other metrics but it is a side effect of the low network load caused by a low PDR. Moreover, ENG-TOT metric is by far the metric with the most route instabilities. So, low PDR values are observed due to a lot of reconfigurations of the DODAG. $\mathrm{R}$ metric achieves better results than all metrics in grid topology with $\mathrm{RX}=100 \%$ since it takes into account the link quality and the residual energy. However, there is a reversal trend in grid topology with $\mathrm{RX}=60 \%$ in which ETX metric gives better results. Maybe it is a consequence of wrong weights affected to energy and link quality parameters in the weighted function of $\mathrm{R}$ metric. Better results might be achieved by the $\mathrm{R}$ metric with weights which are not equal for this two parameters, e.g., 0.25 and 0.75 for energy and link quality respectively. On the whole, ELT and ENGMinMax metrics obtain similar results with a little better results for the former in some cases. We can observe that ELT has a lower PDR than ENG-MinMax metric on grid topology with $\mathrm{RX}=100 \%$, and a better end-to-end delay on grid topology with $\mathrm{RX}=100 \%$ and on random topology with $\mathrm{RX}=60 \%$. These better results are a consequence of the link quality taken into account by the ELT metric contrary to ENG-MinMax metric.

We can notice that we have compared the five considered metrics in a model with a reception ratio of $80 \%$, i.e., a $20 \%$ packet loss. The obtained results are a little better but very similar with the model when $\mathrm{RX}=60 \%$, so due to space constraints these results are not presented in this paper.

\section{CONCLUSION}

To minimise energy consumption, to guarantee a reliable communication and to provide a high delivery ratio is especially challenging in WSN and necessitates to design special mechanisms at the network layer. As a result, RPL was specified by the IETF ROLL working group as a distance vector routing protocol for LLNs. A Destination Oriented Directed Acyclic Graph (DODAG) is constructed by optimizing an objective function which takes into account metrics and constraints for route selection towards the sink.

In this paper, we have presented in grid and random topology the first comparative study of energy-aware metrics that have been proposed to enhance RPL and in particular extend the network lifetime. Moreover, we consider two models for the message reception: a perfect model in which there is no data packet loss $(\mathrm{RX}=100 \%)$, and a more realistic one with a loss of $40 \%$ on data packets $(\mathrm{RX}=60 \%)$. The 


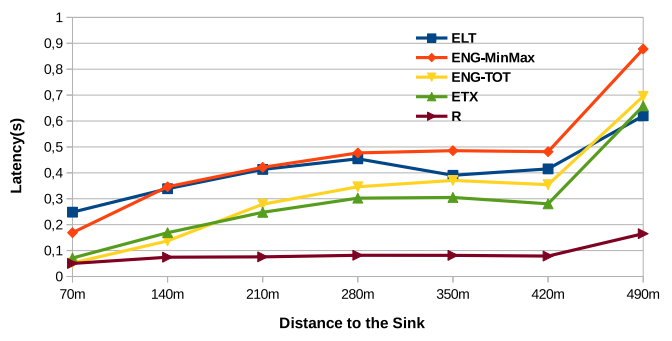

(a) Grid topology with $\mathrm{RX}=100 \%$

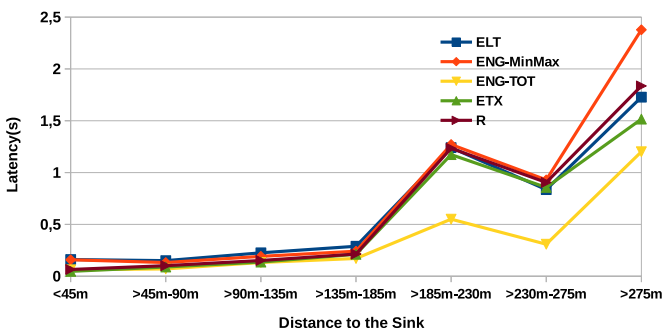

(c) Random topology with $\mathrm{RX}=100 \%$

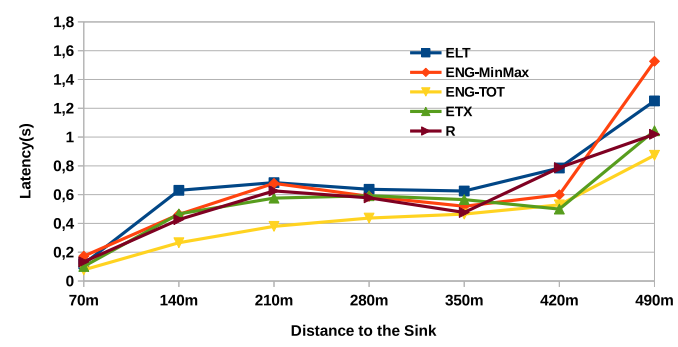

(b) Grid topology with $\mathrm{RX}=60 \%$

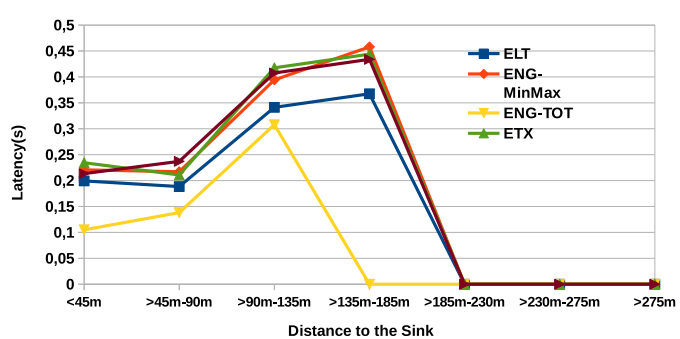

(d) Random topology with $\mathrm{RX}=60 \%$

Figure 4. Latency.

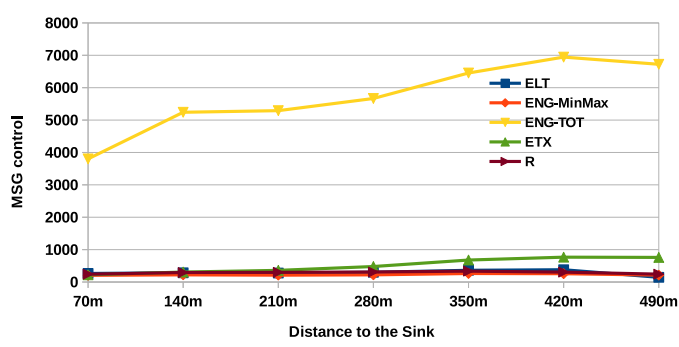

(a) Grid topology with $\mathrm{RX}=100 \%$

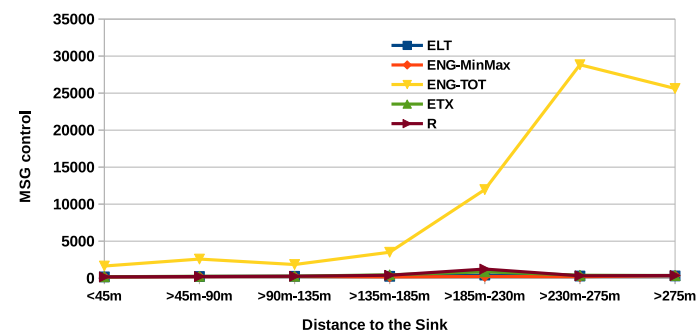

(c) Random topology with $\mathrm{RX}=100 \%$

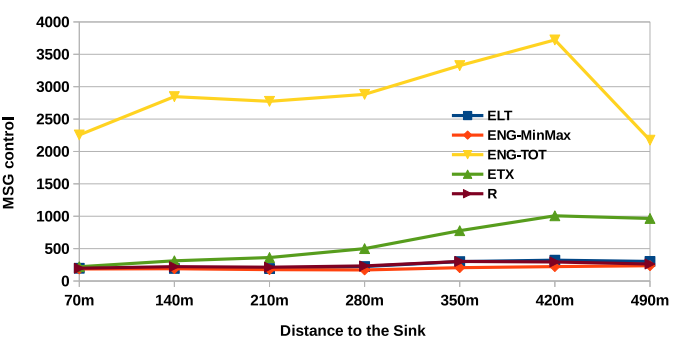

(b) Grid topology with $\mathrm{RX}=60 \%$

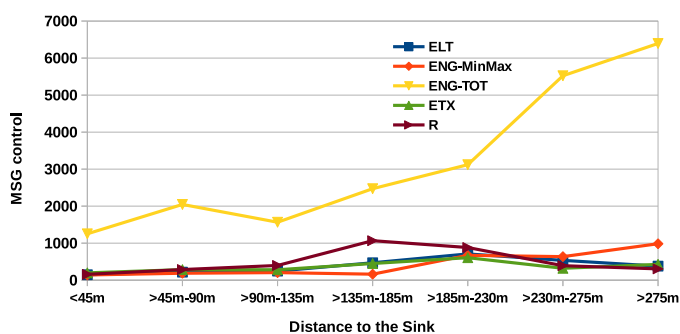

(d) Random topology with $\mathrm{RX}=60 \%$

Figure 5. Control Messages. 
default metric ETX considers the number of retransmissions and allows to reduce indirectly the end-to-end delay towards the sink. However, it reaches a poor network lifetime, despite it reduces the energy consumed for data transmission at each node. The widely used energy-aware RPL metrics achieve better network lifetime, but the end-to-end delays towards the sink may be important. Moreover, it appears that bicriteria metrics such as the $\mathrm{R}$ metric show good performance in terms of network lifetime and end-to-end delays. This can be explained by the fact that the parameters optimized by this metric are not orthogonal. Our results show that there is need for devising multi-criteria metrics that consider both the lossy nature of the link and the low power of the node to improve communication guarantee in WSNs. Some recent works address this aspect by using a general approach but more complex to combine several criteria [20], [21].

\section{REFERENCES}

[1] T. Winter, P. Thubert, A. Brandt, J. Hui, R. Kelsey, P. Levis, K. Pister, R. Struik, J. Vasseur, and R. Alexander, "Rpl: Ipv6 routing protocol for low-power and lossy networks," Internet Requests for Comments, Internet Engineering Task Force (IETF), RFC 6550, 2012. [Online]. Available: https://tools.ietf.org/html/rfc6550

[2] G. Montenegro, N. Kushalnagar, J. Hui, and D. Culler, "Transmission of IPv6 packets over IEEE 802.15.4 networks," Internet proposed standard RFC, vol. 4944, 2007.

[3] M. Qasem, H. Altawssi, M. B. Yassien, and A. Y. AlDubai, "Performance evaluation of RPL objective functions," in 15th IEEE International Conference on Computer and Information Technology; 14th IEEE International Conference on Ubiquitous Computing and Communications; 13th IEEE International Conference on Dependable, Autonomic and Secure Computing; 13th IEEE International Conference on Pervasive Intelligence and Computing, 2015, pp. 1606-1613.

[4] F. Osterlind, A. Dunkels, J. Eriksson, N. Finne, and T. Voigt, "Cross-level sensor network simulation with cooja," in 31st IEEE conference on Local computer networks, 2006, pp. 641648.

[5] O. Gaddour and A. Kouba, "RPL in a nutshell: A survey," Computer Networks, vol. 56, no. 14, pp. 3163-3178, 2012.

[6] F. Demicheli, Design, implementation and Evaluation of an Energy RPL Routing Metric. LAR lambert Academic Publishing, 2014.

[7] G. Xu and G. Lu, "Multipath routing protocol for DAG-based WSNs with mobile sinks," 2nd International Conference on Computer Science and Electronics Engineering (ICCSEE 2013), 2013

[8] P. O. Kamgueu, E. Nataf, T. N. Djotio, and O. Festor, "Energy-based metric for the routing protocol in low-power and lossy network," in 2nd International Conference on Sensor Networks, 2013, pp. 145-148.
[9] R. Draves, J. Padhye, and B. Zill, "Routing in multi-radio, multi-hop wireless mesh networks," in 10th annual international conference on Mobile computing and networking. ACM, 2004, pp. 114-128.

[10] A. Adya, P. Bahl, J. Padhye, A. Wolman, and L. Zhou, "A multi-radio unification protocol for ieee 802.11 wireless networks," in First International Conference on Broadband Networks. IEEE, 2004, pp. 344-354.

[11] C. E. Koksal and H. Balakrishnan, "Quality-aware routing metrics for time-varying wireless mesh networks," IEEE Journal on Selected Areas in Communications, vol. 24, no. 11, pp. 1984-1994, 2006

[12] L.-H. Chang, T.-H. Lee, S.-J. Chen, and C.-Y. Liao, "Energyefficient oriented routing algorithm in wireless sensor networks," in IEEE International Conference on Systems, Man, and Cybernetics (SMC), 2013, pp. 3813-3818.

[13] O. Iova, F. Theoleyre, and T. Noel, "Improving the network lifetime with energy-balancing routing: Application to rpl," in 7th IFIP Wireless and Mobile Networking Conference. IEEE, 2014, pp. 1-8.

[14] O. Gnawali and P. Levis, "The etx objective function for rpl," Internet Engineering Task Force (IETF), Tech. Rep., 2010. [Online]. Available: https://tools.ietf.org/html/draft-gnawaliroll-etxof-00

[15] J. Polastre, R. Szewczyk, and D. E. Culler, "Telos: enabling ultra-low power wireless research," in Fourth International Symposium on Information Processing in Sensor Networks (IPSN), 2005, pp. 364-369. [Online]. Available: http://dx.doi.org/10.1109/IPSN.2005.1440950

[16] A. Dunkels, "The contikimac radio duty cycling protocol," Swedish Institute of Computer Science, Tech. Rep. T2011:13, 2011.

[17] A. Dunkels, J. Eriksson, N. Finne, and N. Tsiftes, "Powertrace: Network-level power profiling for low-power wireless networks," Swedish Institute of Computer Science, Tech. Rep. T2011:05, 2011.

[18] E. Nataf and O. Festor, "Accurate online estimation of battery lifetime for wireless sensors network," in 2nd International Conference on Sensor Networks, 2013, pp. 59-64.

[19] F. Boubekeur, L. Blin, R. Léone, and P. Medagliani, "Bounding degrees on RPL," in 11th ACM Symposium on QoS and Security for Wireless and Mobile Networks, 2015, pp. 123130.

[20] O. Gaddour, A. Koubaa, N. Baccour, and M. Abid, "OF-FL: qos-aware fuzzy logic objective function for the RPL routing protocol," in 12th International Symposium on Modeling and Optimization in Mobile, Ad Hoc, and Wireless Networks, 2014, pp. 365-372.

[21] P. O. Kamgueu, E. Nataf, and T. N. Djotio, "On design and deployment of fuzzy-based metric for routing in low-power and lossy networks," in 40th IEEE Local Computer Networks Conference Workshops, 2015, pp. 789-795. 